\title{
Modeling and Design of an Electrically Actuated Resonant Switch
}

\author{
M. Jrad ${ }^{1,3}$, M.I. Younis ${ }^{2}$, and F. Najar ${ }^{1}$ \\ ${ }^{1}$ Applied Mechanics and Systems Research Laboratory, Tunisia Polytechnic School, University of Carthage, Tunisia. \\ ${ }^{2}$ Binghamton University, State University of New York, USA. \\ ${ }^{3}$ Department of Engineering Science and Mechanics, Virginia Polytechnic Institute and State University, USA.
}

\begin{abstract}
We present modeling and simulation of a new device concept of an electrically actuated resonant switch (EARS), which can be tuned to be triggered at low levels of acceleration, as low as those of earthquakes. The device is realized by mounting an electrostatically actuated cantilever microbeam with a mass at the tip on top of a compliant board or a printed circuit board PCB, which is modeled as a hinged-hinged beam. A distributed-parameter model of the device is derived for the microbeam and the PCB using Hamilton's principle based on the Euler-Bernoulli beam model. The equations are then discretized using the Galerkin procedure. A nonlinear numerical dynamic analysis is performed in order to characterize the behavior and performance of the device when subjected to acceleration pulses. We conduct a parametric study showing several curves of dynamic pull-in threshold for various values of electric voltage loads and frequency of excitations. We show that the device can be triggered at a wide range of acceleration ranging from $0.4 \mathrm{~g}-200 \mathrm{~g}$ for various values of the DC and AC voltages.
\end{abstract}

\section{Introduction}

MEMS technology is rapidly developing due to its compatibility with the manufacturing techniques used in microelectronics. MEMS devices are well commercialized thanks to their low cost. MEMS accelerometers have been under an increasing focus recently for their uses in smart phones, video games, and other consumer electronics. They have been also implemented in a broad range of new applications to sense mechanical shock. Due to their ability to replace complicated systems for sensing with lower cost, they are used in various applications, such as airbag deployment in automobiles and for protection of electronic devices in the case of free fall. Another category of accelerometers is the low- $g$ devices. Such devices suit applications for safety and security, such as for earthquake and oil exploration, by replacing the conventional moving-coil elements used in geophones.

Threshold-accelerometer switches have been studied in the past [1], [2], [3], [4], mostly for their use in airbag applications and weapon arming/disarming. These devices are designed to be triggered when the level of acceleration exceeds certain threshold. This means that they can provide instantaneous action when needed, which is very attractive feature.

Several works have been published studying the reliability of MEMS devices under mechanical shock [5],[6],[7],[8],[9]. Several papers investigated the response of microstructure under the combined effect of electrostatic forces and mechanical shock [5], [7], [9]. These explained the failure of MEMS devices subjected to shock load and showed that a mechanical shock load, even moderate in magnitude, can affect the stability of microstructure electrostatically actuated by reducing its dynamic pull-in, causing therefore an early failure. Younis et al. [5] used a one degree of freedom system, while in [7] Younis et al. used a clamped-clamped beam model. Ouakad et al. [9] presented an experimental investigation of the response of electrostatically actuated microbeams to mechanical shock. In [10], the response of a MEMS resonators when subjected to mechanical shock was investigated.

Many research groups considered the effect of mechanical shock load as undesirable. Their main focus was to limit the impact of such external disturbances. Other groups took profit from this effect to design shock sensors and accelerometers. Younis et al. presented a technique to design smart MEMS switch triggered by acceleration based on capacitive accelerometer [6]. They proved its reliability by showing its independence from pressure. This makes it able to operate under atmospheric condition without need for a special packaging, reducing therefore its cost.

The objective of this paper is to investigate by simulations realizing an electrically actuated resonant switch (EARS), which is tuned to be triggered at low levels of acceleration, as low as those of earthquakes.

\section{Problem Formulation}

We consider a microcantilever beam clamped at one end and with a paddle attached to its tip at the other end, as shown in Fig. 1. Both assembly parts are assumed to be made of a single structural layer of polysilicon. The 
paddle is actuated by an electrode attached to the substrate at a distance $d$ underneath the mass. The microbeam is modeled as an Euler-Bernoulli beam of width $b_{11}$, thickness $h_{1}$, cross-sectional area $A_{1}=b_{11} h_{1}$, and second moment of area $I_{1}=b_{11} h_{1}{ }^{3} / 12$. The paddle is modeled as a rigid body with mass $M$, mass moment of inertia $J_{1}=M_{1} L_{\mathrm{c}}{ }^{2} / 3$, and center of mass lying at a distance $L_{c}$ along the center line from the beam tip. The location of the paddle center of mass $C$ is determined by its position $w_{1 c}=w_{1}\left(x_{c}, t\right)$ and its orientation about the horizontal axis $\theta_{c}$, Fig. 2 .

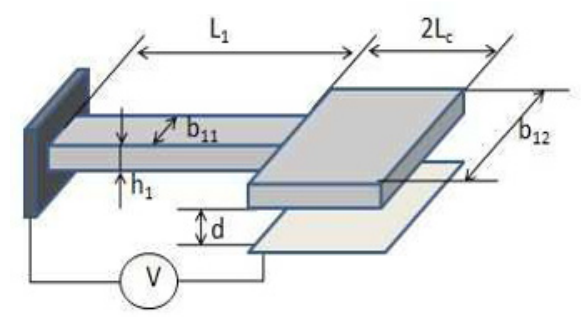

Fig. 1 Schematic of a microcantilever beam.

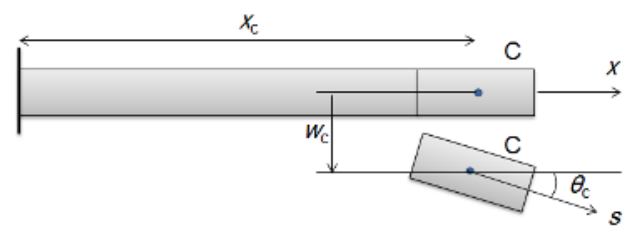

Fig. 2 Local coordinate system attached to the paddle.

The cantilever microbeam is attached from its fixed end to a complaint PCB, which is modeled as a hingedhinged beam (Fig. 3). The assembly constitutes the proposed device in this paper.

Next, we derive the equations of motion of the system using the Hamliton's Principle. For that, we calculate the kinetic and potential energies and assume that the PCB's dimensions are much larger than those of the microcantilever beam. Hence, the microcantilever beam has no effect on the hinged-hinged substrate beam. However, the microcantilever is affected by the PCB motion.

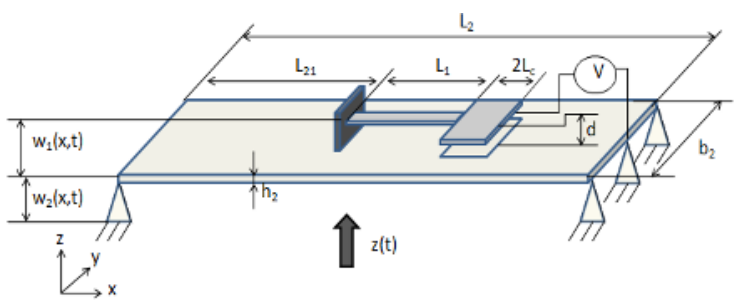

Fig. 3 Schematic of the device components.

In what follows the subscript 1 is used for the upper beam, and the subscript 2 is used for the lower beam. The deflection is denoted by $w$. The applied voltage between the two electrodes of the cantilever microbeam is given by $V=V_{D C}+V_{A C} \operatorname{Cos}(\Omega t)$.

The application of the Hamilton's principle generates a set of two equations of motion and the corresponding 8 boundary conditions.

$\rho_{1} A_{1}\left(\ddot{w}_{1}+\ddot{w}_{2}\left(L_{21}, t\right)+\ddot{z}\right)+c_{1} \dot{w}_{1}+E_{1} I_{1} w^{I V}{ }_{1}=0$ For $: x \in\left[0, L_{1}\right]$ $\rho_{2} A_{2}\left(\ddot{w}_{2}+\ddot{z}\right)+c_{2} \dot{w}_{2}+E_{2} I_{2} w_{2}^{I V}=0$ For $: x \in\left[0, L_{2}\right]$ and associated boundary conditions

$$
\begin{aligned}
& w_{1}(0, t)=0, \quad w_{1}^{\prime}(0, t)=0, \\
& w_{2}(0, t)=w_{2}\left(L_{2}, t\right)=0, \quad w_{2}^{\prime \prime}(0, t)=w_{2}^{\prime \prime}\left(L_{2}, t\right)=0, \\
& E_{1} I_{1} w^{\prime \prime}{ }_{1}\left(L_{1}, t\right)=M_{1}\left(\ddot{w}_{1}\left(L_{1}, t\right)+\ddot{w}_{2}\left(L_{21}, t\right)+\ddot{z}(t)\right. \\
&+\left.L_{c}\left(\ddot{w}_{1}^{\prime}\left(L_{1}, t\right)+\ddot{w}_{2}^{\prime}\left(L_{21}, t\right)\right)\right) \\
&-\frac{\varepsilon b_{12} L_{c} V_{D C}^{2}}{\left(d-w_{1}\left(L_{1}, t\right)\right)\left(d-w_{1}\left(L_{1}, t\right)-2 L_{c} w_{1}^{\prime}\left(L_{1}, t\right)\right)} \\
& E_{1} I_{1} w_{1}{ }_{1}^{\prime}\left(L_{1}, t\right)=-M_{1} L_{c}\left(\ddot{w}_{1}\left(L_{1}, t\right)+\ddot{w}_{2}\left(L_{21}, t\right)+\ddot{z}(t)\right. \\
&\left.+L_{c}\left(\ddot{w}_{1}^{\prime}\left(L_{1}, t\right)+\ddot{w}_{2}^{\prime}\left(L_{21}, t\right)\right)\right) \\
&-J_{1}\left(\ddot{w}_{1}^{\prime}\left(L_{1}, t\right)+\ddot{w}_{2}^{\prime}\left(L_{21}, t\right)\right) \\
&+\frac{\varepsilon b_{12} V_{D C}^{2}}{2\left(w_{1}^{\prime}\left(L_{1}, t\right)\right)^{2}}\left[\frac{2 L_{c} w_{1}^{\prime}\left(L_{1}, t\right)}{d-w_{1}\left(L_{1}, t\right)-2 L_{c} w_{1}^{\prime}\left(L_{1}, t\right)}\right. \\
&\left.+\log \left[\frac{d-w_{1}\left(L_{1}, t\right)-2 L_{c} w}{d-w_{1}\left(L_{1}, t\right)}\right]\right]
\end{aligned}
$$

The input acceleration applied to the system is received by the lower beam as a half-sine base excitation (3), where $A_{s h}$ and $T_{s h}$ are, respectively, its amplitude and period and $H(\mathrm{t})$ is the Heaviside step function. The applied acceleration is then transmitted to the upper microbeam through the fixed side via the term $\ddot{w}_{2}\left(L_{21}, t\right)+\ddot{z}(t)$.

$$
\ddot{z}(t)=A_{s h}\left(\begin{array}{l}
H(t) \operatorname{Sin}\left(\frac{\pi}{T_{s h}} t\right) \\
+H\left(t-T_{s h}\right) \operatorname{Sin}\left(\frac{\pi}{T_{s h}}\left(t-T_{s h}\right)\right)
\end{array}\right)
$$

(3)

\section{Dynamic Analysis}

Table 1 Geometric and physical parameters of the microcantilever beam and the paddle.

\begin{tabular}{|c|c|c|c|c|}
\hline$L_{1}$ & $b_{11}$ & $h_{1}$ & $d$ & $L_{\mathrm{c}}$ \\
\hline $400 \mu \mathrm{m}$ & $50 \mu \mathrm{m}$ & $4 \mu \mathrm{m}$ & $2 \mu \mathrm{m}$ & $30 \mu \mathrm{m}$ \\
\hline$b_{12}$ & $\rho_{1}$ & $E_{1}$ & $\varepsilon$ & $\xi$ \\
\hline $75 \mu \mathrm{m}$ & $2332 \mathrm{Kg}$ & $160 \mathrm{GPa}$ & $8.854 \times 10^{-12}$ & $1 / 300$ \\
\hline
\end{tabular}

Table 2 Geometric and physical parameters of the hingedhinged beam.

\begin{tabular}{|c|c|c|c|c|c|}
\hline$L_{2}$ & $b_{2}$ & $h_{2}$ & $\rho_{2}$ & $E_{2}$ & $\xi$ \\
$2 \mathrm{~cm}$ & $0.5 \mathrm{~cm}$ & $200 \mu \mathrm{m}$ & $2700 \mathrm{Kg}$ & $70 \mathrm{GPa}$ & $1 / 100$ \\
\hline
\end{tabular}

We consider as a case study the system of geometric and physical parameters of Table 1 and Table 2. After putting the system of equations (1) and (2) into dimensionless form, we solve for the static deflection $w_{\mathrm{s}}(x)$ (of the upper beam) by dropping the time depending terms and assuming steady solution. Then, using the same equations, we approximate the deflection with $w(x, t)=$ $w_{s}(x)+\varphi(x) e^{i \omega t}$, linearized the equation, and solve the eigenvalue problem for the frequencies $\omega$ and mode shapes $\varphi(x)$. The first natural frequencies, of the associated linearized and undamped eigenvalue problem to equations 1 and 2, are given in Table 3. Where the subscript $L$ indicates the natural frequencies of the lower 
hinged-hinged beam, and the subscript $U$ indicates those of the upper microcantilever beam.

In addition, the static analysis of the system indicates that the pull-in voltage of the upper microcantilever beam is located around $V_{D C}=9.8 \mathrm{~V}$.

Table 3 Natural frequencies of the system.

\begin{tabular}{|ccccc|}
\hline$\omega_{I L}$ & $\omega_{2 L}$ & $\omega_{3 L}$ & $\omega_{1 U}$ & $\omega_{2 U}$ \\
$1.15 \mathrm{kHz}$ & $4.62 \mathrm{KHz}$ & $10.39 \mathrm{kHz}$ & $22.93 \mathrm{kHz}$ & $150.7 \mathrm{kHz}$ \\
\hline
\end{tabular}

In order to investigate the performance of the proposed EARS, we examine the variation of the threshold instability, which is the lowest detectable acceleration (LDA), at which the microbeam collapses as a switch $\ddot{Z}(t)$, for a fixed frequency $\Omega$ (the amplitude $A_{s h}$ is varied). For this, we solve the equations of motion, equations (1) and (2), using, for example, a Galerkin procedure and the mode shape of the associated undamped and unforced eigenvalue problem as test functions. We vary the acceleration level with two preselected excitation frequencies $\left(\Omega=0.7 \omega_{1 L}\right.$ and $\left.\Omega=\omega_{1 L}\right)$ and solve for the displacements $w_{1}$ and $w_{2}$. We then detect if the upper microcantilever beam collapse on to the substrate through pull-in instability (Fig. 4). This will give the LDA by the proposed design.



(a) $A_{\mathrm{sh}}<\mathrm{LDA}$

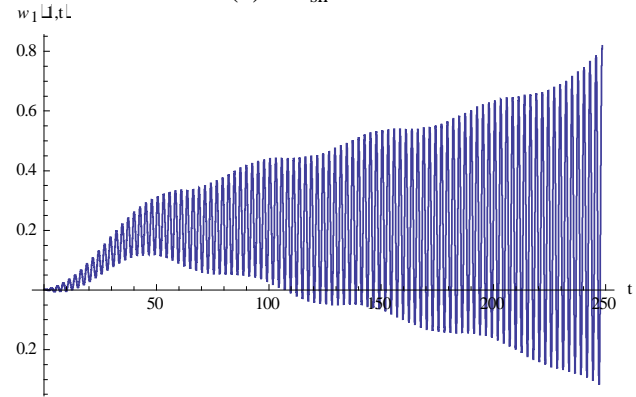

(b) $A_{\mathrm{sh}}>\mathrm{LDA}$

Fig. 4 Time history of the tip of the microbeam showing the stable (a) and unstable (b) motion of the device for two different input accelerations.

In Fig. 5, we show the variation of the LDA for different DC and AC voltages when $\Omega=0.7 \omega_{1 L}$. In this case, we notice that the LDA decreases with both DC and $\mathrm{AC}$ voltages. As an example, under an applied AC voltage of $1 \mathrm{~V}$, the sensitivity of the device is doubled if we increase the DC voltage from 2 to $6 \mathrm{~V}$. Similarly, if we fix the static voltage to $2 \mathrm{~V}$, the device is able to sense about $100 \mathrm{~g}$ for an $\mathrm{AC}$ of $1 \mathrm{~V}$. Nonetheless, if we double the $\mathrm{AC}$ voltage, the device becomes more sensitive by detecting a lower acceleration of $70 \mathrm{~g}$. In fact, by increasing the applied voltage, the microcantilever's first frequency decreases [11], which means that its effective stiffness also decreases leading to an earlier collapse of the microcantilever beam through a dynamic pull-in. As a result, the device becomes more sensitive to external acceleration.

We mention that the LDA behaves linearly with the DC voltage for $\Omega / \omega_{1 L}=0.7$ and most generally for ratios far from 1 [8]. Next we focus on ratios close to 1 . Since the objective of the present work is to increase the sensitivity of EARS and try to enable detection of acceleration less than $1 g$ only by tuning the electrostatic force. Although we have improved the sensitivity in this case, the result is still far from the desired low- $g$ range.

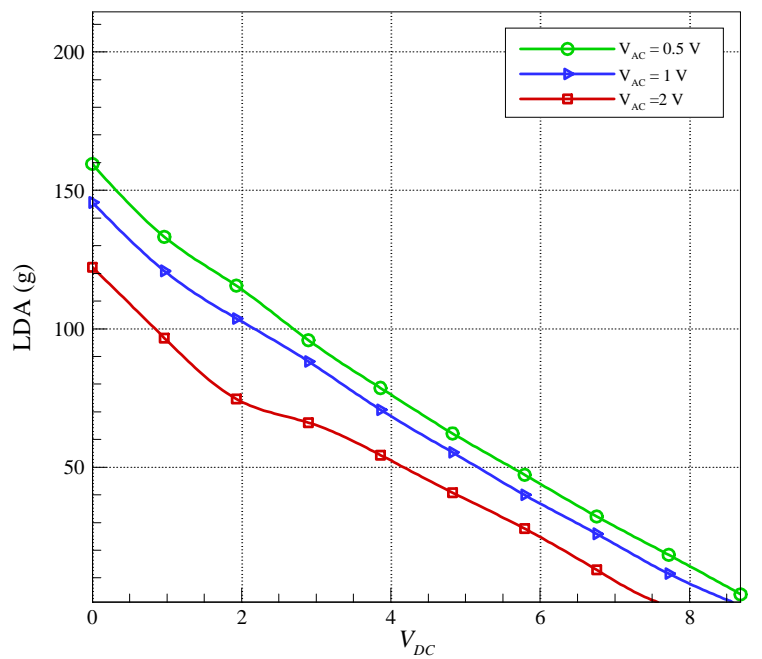

Fig. 5 Variation of the threshold instability with $V_{D C}$ for an excitation frequency of $0.7 \omega_{1 L}, T_{s h}=1 \mathrm{~ms}$, and various $V_{A C}$.

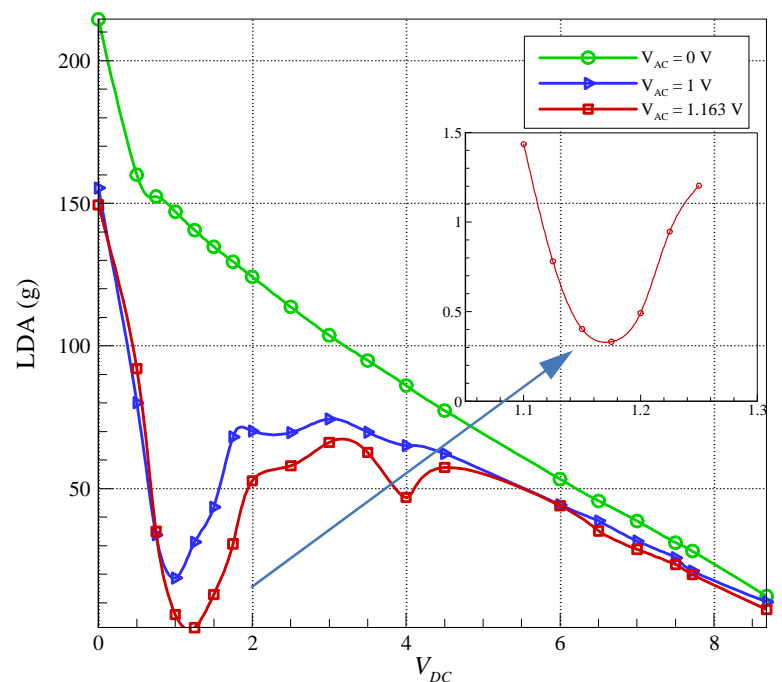

Fig. 6 Variation of the threshold instability for $\Omega=\omega_{1 \mathrm{~L}}$ and $\mathrm{T}_{\mathrm{sh}}=1 \mathrm{~ms}$.

For the second case, we operate at resonance $(\Omega=$ $\left.\omega_{1 L}\right)$. The resulting LDA performances as a function of the applied voltages are shown in Fig. 6. For $V_{\mathrm{AC}}=0 V$, the shape of the curve is the same as in Fig. 5. However, we notice that the shape of the curve is no longer linear for the two other AC voltages ( $1 V$ and $1.163 V$ ). This is shown clearly by the creation of a finger-shaped curve $(0.5-3 \mathrm{~V})$ where the LDA is much lower than in the linear case. Let us call this band of voltage the band of 
weakness of the structure. The new finger-shaped curve presents a minimum of LDA where we can get the best sensitivity with less power. For example, the optimum value of $\mathrm{DC}$ for the case $\mathrm{V}_{\mathrm{AC}}=1 \mathrm{~V}$ is $\mathrm{V}_{\mathrm{DC}}=1 \mathrm{~V}$ and the associated LDA is $20 \mathrm{~g}$. We conclude from Fig. 6 that if we operate close to resonance, a band of weakness of the structure is created and we can obtain a higher sensitivity by selecting the optimal value of DC voltage. It should be noted here that we improved the sensitivity by reducing the LDA from around $1500 \mathrm{~g}$ to less than $0.4 \mathrm{~g}$. The sudden decrease in the LDA and then increase can be justified by noting that for some range of $V_{\mathrm{DC}}, \Omega=\omega_{I L}$ represents an operating point on the maximum of the frequency-response curve. As $V_{\mathrm{DC}}$ increases more, the natural frequency of the cantilever decreases, due to the softening effect. This means, $\Omega=\omega_{1 L}$ no longer represents the operating point of the maximum response of the EARS, but rather a reduced value of $\Omega=\omega_{1 L}$. For example, as shown in Fig. 6, for some range of $V_{\mathrm{DC}}$, various reduced values of $\Omega=\omega_{I L}$ yield the optimal operating frequency of the EARS where the response is maximum, and hence LDA is minimum.

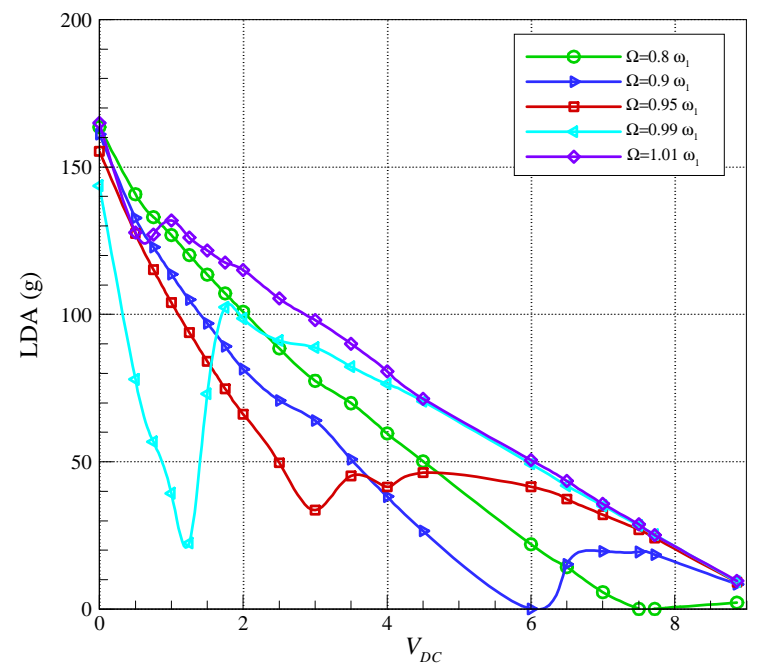

Fig. 7 Variation of the LDA with $\mathrm{V}_{\mathrm{DC}}$ for $\mathrm{V}_{\mathrm{AC}}=0.5 \mathrm{~V}, \mathrm{~T}_{\mathrm{sh}}=1 \mathrm{~ms}$ and various excitation frequencies.

In what follows, we are interested in the behavior of the band of weakness of the structure. We show in Fig. 7 the LDA- $V_{D C}$ curve for different excitation frequencies. By sweeping the excitation frequency from $0.8 \omega_{1 L}$ to $1.01 \omega_{1 L}$ we notice that the curve LDA- $V_{D C}$ is initially linear. A small band of weakness is created for $\Omega=$ $0.9 \omega_{1 \mathrm{~L}}(4.5-7 \mathrm{~V})$. It is then moving backward, as we sweep the frequency, and the minimum valued of LDA increases. After reaching $\Omega=1.01 \omega_{1 \mathrm{~L}}$, the nonlinearity disappears and the curve LDA- $V_{D C}$ returns to its original shape. To conclude, sweeping the excitation frequency gives an idea of the variation of the sensitivity of the device with the electrostatic parameters and allows choosing the optimized configuration that gives a device of high performance consuming, relatively, low voltage load.

\section{Conclusion}

In this work, an investigation of the response of a resonant accelerometer was presented. Several results have shown that we can enhance the sensitivity of the device by increasing the electrostatic load. However, we showed that operating the system close to the primary resonance creates nonlinearity in the relationship LDA$V_{D C}$ that can be used to improve the sensitivity of the device and reduce the LDA from about $1500 \mathrm{~g}$ up to $0.4 \mathrm{~g}$. By sweeping the excitation frequency we showed that a band of voltage appears close to resonance where the structure becomes weaker and, thus, more sensitive to input acceleration. Therefore, an optimization process is needed to select the appropriate parameters that give higher sensitivity sensors requiring, relatively, low power.

\section{References}

1. Frobenius, W.D., et al., Microminiature ganged threshold accelerometers compatible with integrated circuit technology. Electron Devices, IEEE Transactions on, (1972). 19(1): p. 37-40.

2. Robinson C, et al., Problem encountered In The development of the microscale G-switch using three design approaches in Proc. Int. Conf. on Solid-State Sensors and Actuators (1987) Tokyo, Japan. p. 410413.

3. Loke, Y., G.H. McKinnon, and M.J. Brett, Fabrication and characterization of silicon micromachined threshold accelerometers. Sensors and Actuators A: Physical, (1991). 29(3): p. 235-240.

4. Man, P.F. and C.H. Mastrangelo. Surface micromachined shock sensor for impact detection. In Proc. Solid-State Sensor and Actuator Work shop. (1994). Hilton Head, South Carolina.

5. Mohammad I Younis, R.M.a.D.J., Investigation of the response of microstructures under the combined effect of mechanical shock and electrostatic forces Journal of Micromechanics and Microengineering, (2006). 16(11): p. 2463-2474.

6. Younis, M.I., et al., Characterization of the performance of capacitive switches activated by mechanical shock. Journal of Micromechanics and Microengineering, (2007). 17(7): p. 1360.

7. Younis, M.I., F. Alsaleem, and D. Jordy, The response of clamped-clamped microbeams under mechanical shock. International Journal of Non-Linear Mechanics, (2007). 42(4): p. 643-657.

8. Alsaleem, F., M.I. Younis, and R. Miles, An Investigation Into the Effect of the PCB Motion on the Dynamic Response of MEMS Devices Under Mechanical Shock Loads. Journal of Electronic Packaging, (2008). 130(3): p. 031002-10.

9. Ouakad, H., et al. Response of an electrostatically actuated microbeam to drop-table test. in Thermal, Mechanical \& Multi-Physics Simulation, and Experiments in Microelectronics and Microsystems (EuroSimE), 11th International Conference (2010).

10. Ibrahim, M.I. and M.I. Younis, The dynamic response of electrostatically driven resonators under 
CSNDD 2012

mechanical shock. Journal of Micromechanics and Microengineering, (2010). 20(2): p. 025006.

11. Nayfeh, A.H., et al., Nonlinear dynamics of a resonant gas sensor Nonlinear Dynamics, (2009). 59(4): p. 12. 